\title{
A common mechanism behind distractor-response and response-effect binding?
}

\author{
Birte Moeller $^{1} \cdot$ Roland Pfister $^{2} \cdot$ Wilfried Kunde $^{2} \cdot$ Christian Frings $^{1}$
}

Published online: 25 January 2016

(C) The Psychonomic Society, Inc. 2016

\begin{abstract}
Short-term bindings between responses and events in the environment ensure efficient behavioral control. This notion holds true for two particular types of binding: bindings between responses and response-irrelevant distractor stimuli that are present at the time of responding, and also for bindings between responses and the effects they cause. Although both types of binding have been extensively studied in the past, little is known about their interrelation. In three experiments, we analyzed both types of binding processes in a distractorresponse binding design and in a response-effect binding design, which yielded two central findings: (1) Distractorresponse binding and response-effect binding effects were observed not only in their native, but also in the corresponding "non-native" design, and (2) a manipulation of retrieval delay affected both types of bindings in a similar way. We suggest that a general and unselective mechanism is responsible for integrating own responses with a large variety of stimuli.
\end{abstract}

Keywords Cognitive control and automaticity · Perception and Action

Birte Moeller

moellerb@uni-trier.de

1 Department of Psychology, University of Trier, Universitätsring 15, D-54296 Trier, Germany

2 Department of Psychology, University of Würzburg, Röntgenring 11, D-97070 Würzburg, Germany

\section{Introduction}

Human action control is astonishingly successful in the large majority of cases, even though it has to deal with a continuously changing environment. Most of us take this efficiency for granted, yet this convenience is only possible because cognitive processes continuously extract information from the environment. Any natural environment comes with a certain structure in terms of events that are likely to go together and others that are not. Given these abundant contingencies between different events, rapid acquisition and usage of knowledge about them helps successful behavior in stable and changing environments alike. Indeed, a large number of studies provide evidence that contingencies between (response-irrelevant) stimuli and responses, and between responses and effects can be learned quickly and incidentally (Garner \& Felfoldy, 1970; Logan, 1988, 1990; Miller, 1987; Nissen \& Bullemer, 1987; Schmidt, Crump, Cheesman, \& Besner, 2007). While many studies investigating the usage of environmental contingencies in action control focused on long-term effects of learning processes, the first step in a learning process - that is, the very first encounter of a particular contingency - could be considered the smallest learning unit which might become reinforced by recurrence. In this regard the investigation of short-term associations between stimuli, responses, and following effects provides insight into the mechanisms that ensure behavioral efficiency in a variety of tasks (Dutzi \& Hommel, 2009; Giesen \& Rothermund, 2011; Hommel, Proctor, \& Vu, 2004; Mayr \& Buchner, 2006; Moeller \& Frings, 2011; Tipper, 1985).

Not surprisingly, different traditions of psychological theorizing have elaborated on the processes underlying the rapid formation of associations between responses and events in an agent's surroundings at their very first encounter. In the following, we will describe two of these traditions in more detail, 
both of which focus on short-term associations that are acquired incidentally. One of these traditions focuses on the formation of action plans and the influence, recently used action plans can have on current behavior (Frings, Rothermund, \& Wentura, 2007; Frings \& Moeller, 2010; Hommel, 1998; Mayr \& Buchner, 2006), whereas the other tradition focuses on the role of action-contingent changes in the environment and how these action effects are used for action control (Elsner \& Hommel, 2001; Dutzi \& Hommel, 2009; Kunde, 2001; Pfister, Kiesel, \& Melcher, 2010).

\section{Binding responses and concurrent distractor stimuli}

Actions are typically not performed in a barren, deprived environment but rather in the presence of various stimuli that are unrelated to the task at hand. Several cognitive mechanisms have been shown to deal with these distractor stimuli to ensure adequate and efficient responding (Neill, 1997; Tipper, 1985; Treisman, 1960). For example, cumulative evidence suggests that features of such distractor stimuli are automatically integrated into action plans and linked to responses that are performed in their presence. The resulting association is typically labeled distractor-response binding (Frings et al., 2007; Rothermund et al., 2005). Distractor-response bindings have been documented after the very first encounter with a distractor stimulus and are likely the first step in an incidental association learning process (Moeller \& Frings, 2014a). After response execution, these bindings survive for a brief period of time (approximately 1 second, see Frings, 2011). During this time, the association is reactivated if any part of it reoccurs. Hence, repeating a stimulus that was earlier integrated in an action plan leads to response facilitation if the same response has to be repeated and, conversely, it leads to response impairment if a different response is required. Research on stimulus-response bindings in general has uncovered a wide variety of stimulus-response associations that are integrated automatically (Frings et al., 2007; Frings, Moeller, \& Rothermund, 2013; Hommel, 1998, 2004; Hommel et al., 2004; Horner \& Henson, 2009, 2011; Mayr \& Buchner, 2006; Logan, 1988, 1990; Waszak, Hommel, \& Allport, 2004, for a recent review on stimulus-response binding in general, see Henson, Eckstein, Waszak, Frings, \& Horner, 2014). The fact that not only currently relevant information is associated to own responses, but also additional response-irrelevant features, indicates that action plans readily accommodate any kind of environmental contingency.

Integration of response-irrelevant stimuli into action plans can be investigated by analyzing performance in a primeprobe design as a function of the corresponding response sequence and distractor sequence. In a typical paradigm to probe for distractor-response binding (Frings et al., 2007), participants respond to a target stimulus while ignoring a distractor, both on the prime and on the probe. Response relation (repetition vs. change from prime to probe) is varied orthogonally to distractor relation (repetition vs. change). Distractorresponse binding is indicated by an interaction of response relation and distractor relation for probe reaction times (RTs) and error rates: Distractor repetition facilitates responding if the response has to be repeated but tends to hamper performance if different responses are required to the prime and the probe target. In other words, a difference between distractor repetition effects for response repetition and response change trials indicates binding between distractor stimulus and response. This procedure thus offers a straightforward measure for short-term bindings between irrelevant information and own responses.

\section{Binding responses and subsequent effects}

Environmental contingencies do not only apply to stimuli that are present before or concurrently with a response, but also between responses and their perceivable consequences, i.e., effects. In fact, knowing about contingencies between actions and subsequent changes in the environment is inevitable for adaptive decision making (Botvinick, 2007; Cohen, McClure, \& Yu, 2007; Rescorla \& Wagner, 1972). In addition to biasing decisions, anticipations of to-be-produced changes in the environment also serve more basic functions for human action control. Such a central, generative role of anticipative processes is emphasized by effect-based theories of human motor control (Shin, Proctor, \& Capaldi, 2010). These theories claim that overt body movements are initiated and controlled by anticipations of the effects they produce. Early formulations of effect-based theories thereby focused on body-related changes in terms of the visual and proprioceptive reafferences produced by the moving body (Pfister, Janczyk, Gressmann, Fournier, \& Kunde, 2014). In other words, raising one's arm can only be achieved by anticipating how the raising limb would look and feel like. This focus on body-related reafferences was challenged by empirical findings across the last decades that indicated any type of action-contingent change to have the potential for being included in motor control (Hommel, 1993; Kunde, 2001, 2003; Hubbard, Gazzaley, \& Morsella, 2011; Pfister, Dignath, Hommel, \& Kunde, 2013; Pfister, Pfeuffer, \& Kunde, 2014).

Before being able to utilize action-contingent effects for motor control, agents need to acquire associations between own responses and following effects (Elsner \& Hommel, 2001, 2004; Herwig \& Horstmann, 2011; Herwig \& Waszak, 2009; Hoffmann, Lenhard, Sebald, \& Pfister, 2009; Hommel, Alonso, \& Fuentes, 2003; Sato \& Itakura, 2013). This learning process obviously also begins with the very first episode of a particular response causing a particular effect (Dutzi \& Hommel, 2009). Studies on this microgenesis of response-effect associations focus on aftereffects of individual responses and typically use a simple experimental procedure: 
Participants are given the opportunity to perform one or another response and each response triggers a particular effect (e.g., a left button press triggering a high-pitched sound). In a second part of each trial, a stimulus appears that either resembles the previous effect (high-pitched sound) or not (lowpitched sound). Participants are to decide among the two responses again and their choice can be effect-consistent (in the example this would mean a left button press for sound repetition and a right button press for sound change) or effectinconsistent (right button in case of sound repetition and left button in case of sound change). An above-chance preference for effect-consistent choices is typically interpreted as reflecting a short-term binding between action and following effect (Dutzi \& Hommel, 2009; Herwig \& Waszak, 2012; Janczyk, Heinemann, \& Pfister, 2012).

\section{One binding mechanism or two?}

The processes of distractor-response binding and responseeffect binding reviewed above allow for representing a wide variety of contingencies in the environment. A better insight into mechanisms responsible for these binding processes therefore has the potential to broaden the general understanding of human behavioral flexibility in many situations. Even though the two processes seem to differ in several aspects and have been investigated using different measures (RTs vs. choice frequencies), both effects have been accounted for within the same theoretical framework of the theory of event coding (TEC, Hommel, 2009; Hommel, Müsseler, Aschersleben, \& Prinz, 2001; see Frings \& Rothermund, 2011, for distractor-response binding, and Dutzi \& Hommel, 2009, for response-effect binding). That is, both distractorresponse binding and response-effect binding are typically assumed to result from mechanisms at work during the creation of event files (Hommel, 2004).

Because both distractor-response and response-effect bindings can become part of the same representational structure (i.e., event files), it seems likely that the effects resemble each other (Dutzi \& Hommel, 2009). In fact, binding has been suggested to be a rather unselective mechanism that binds any stimuli available at the time of responding (for a corresponding theoretical position, see Henson et al., 2014). This suggests that the two types of binding result from the very same process, and the current experiments set out to gather first empirical evidence for implications that follow from this hypothesis.

Yet, there also is good reason to believe that distractorresponse binding and response-effect binding are mediated by different mechanisms. For instance, it has been assumed that learning can be described in hierarchical terms, suggesting a precedence of response-effect learning, and only in a second step response-effect associations to be associated with context or stimulus information (Rescorla, 1991). In line with this theoretical notion, response-effect associations indeed seem to be formed more readily than stimulus-response associations when pitted directly against each other (Hoffmann \& Sebald, 2000; Stock \& Hoffmann, 2002). Acquiring associations between target symbols and responses was found to be more difficult than between responses and effect symbols, even if contingencies between symbols and responses favored learning of stimulus-response associations over responseeffect associations (Stock \& Hoffmann, 2002). Assuming that this difference is already present for the very first encounter of a particular contingency suggests that distractor-response binding and response-effect binding might differ at least quantitatively.

Even a qualitative difference seems possible when considering the phenomenological consequences that are associated with experiencing an effect to occur after own responses as compared to experiencing a distractor to interfere during action planning. A remarkable epiphenomenon of causing an effect in the environment is the feeling of subjective agency, i.e., the characteristic feeling of being causally responsible for the ensuing events (Haggard \& Tsakiris, 2009). Furthermore, having associated an effect with an own response affects perception of this particular effect. More precisely, the phenomenological representation of an effect was found to be attenuated when the effect was experienced while the agent currently planned to execute the associated action (Pfister, Heinemann, Kiesel, Thomaschke, \& Janczyk, 2012) or right after having executed this action (Cardoso-Leite, Mamassian, SchützBosbach, \& Waszak, 2010). Such findings, typically labelled as action-induced blindness or sensory attenuation, have been attributed to response effects that are represented in an event file (Hommel \& Müsseler, 2006; Müsseler \& Hommel, 1997; Thomaschke, Hopkins, \& Miall, 2012). These phenomenological consequences seem to be specific for the representation of anticipated effects of own responses (Müsseler, Wühr, Danielmeier, \& Zysset, 2005; but see Stevanovski, Oriet, \& Jolicoeur, 2006) and therefore suggest that response-effect binding on the one hand and distractor-response binding on the other hand might indeed differ qualitatively.

\section{Current study}

The current study was designed to test implications of the notion that bindings between distractors and responses and between responses and effects can be accounted for by the same mechanism. In two experiments, we directly compared bindings between task-irrelevant distractor stimuli and responses versus responses and task-irrelevant effects. To this end, we adjusted the two paradigms that have been used to investigate distractor-response binding and response-effectbinding to measure each effect in its native design as well as in the corresponding non-native design. Experiment 1 probed for distractor-response bindings in a design akin to those used 
for studying short-term, response-effect associations (while at the same time assessing these associations for comparison), whereas Experiment 2 probed for response-effect bindings in a design akin to those used for studying distractor-response bindings. In a third experiment, we analyzed the effect of varying the interval between binding and retrieval for each binding type.

A first critical question was whether each binding effect also would occur in its non-native design; that is, Experiment 1 asked whether distractor-response binding also would be evident in choice rates when freely choosing between two responses in the face of a repeated versus changed distractor. Conversely, Experiment 2 asked whether shortterm response-effect binding would be evident in a choice RT task when previous effects occur as distractors in this task. A second question targeted whether both binding effects would be influenced when actively manipulating the delay between binding and retrieval. In short, (a) we consistently observed each binding effect in its non-native design, the two binding effects were correlated across participants, and (b) both were affected by retrieval delay.

\section{Experiment 1}

In Experiment 1, we probed for distractor-response bindings in a design akin to those used for studying short-term response-effect bindings (Dutzi \& Hommel, 2009; Herwig \& Waszak, 2012; Janczyk et al. 2012). To this end, we used a prime-probe sequence with a forced choice task for the prime display and a free choice response in the probe display (Fig. 1a).

In effect blocks, each prime response triggered an auditory effect (a high- or low-pitched sound). The probe stimulus of the following free choice response also was a high- or lowpitched sound that could either resemble the previous effect or an alternative sound. Following previous studies with similar setups (Dutzi \& Hommel, 2009; Janczyk et al., 2012), we expected participants to choose response repetitions more often when the sound repeated than when it changed from prime to probe. Distractor blocks were identical to effect blocks, except that a distractor sound accompanied each prime target and prime responses did not produce any auditory effects.

\section{Method}

Participants Twenty students (16 females) from the University of Trier took part in the experiment. Their median age was 20 (range 18-24) years. Participants received course credit or monetary compensation.

Three participants had to be replaced, because they reported to have used response strategies in the probe responses (e.g., switching response side every other response or always repeating the prime response). Two other participants who did not follow instructions (responded with only one hand, or always tried to press both keys simultaneously during free choice) and one participant who responded extremely slowly in prime trials (mean RT more than 1.5 interquartile ranges above the third quartile of the remaining sample in both blocks) were replaced, as well. Finally, we also replaced all participants who repeated the response from the prime in the probe in more than $90 \%$ or less than $10 \%$ of the trials in either of the experimental blocks, which we interpreted as a strong indication of a general response repetition or switch strategy. This criterion applied to three further participants. Including the replaced participants in the analyses did not substantially alter the results.

Materials The experiment was conducted using the E-prime software (E-prime 2.0). Instructions and target stimuli were shown in white on black background (luminance level $0.2 \mathrm{~cd} / \mathrm{m}^{2}$ ) on a standard CRT screen. Target stimuli were the letters D, F, J, and K. They had a horizontal visual angle of $0.8^{\circ}$ to $1.1^{\circ}$ and a vertical visual angle of $0.9^{\circ}$ to $1.0^{\circ}$ and luminance levels of 45 to $75 \mathrm{~cd} / \mathrm{m}^{2}$. Auditory (distractor and effect) stimuli were two $300 \mathrm{~ms}$ and $80 \mathrm{~dB}$ sine wave sounds of 400 and $800 \mathrm{~Hz}$ respectively. Viewing distance was approximately $60 \mathrm{~cm}$.

Procedure Participants were tested individually in soundproof chambers. Instructions were given on the screen and summarized by the experimenter. Participants were instructed to place their left index finger on the key $\mathrm{D}$ and their right index finger on the key K of a standard QWERTZ keyboard. Their task was always to classify the target letter in the prime display by pressing a key with the corresponding finger and ignore any sound that could be presented as a distractor simultaneously with the target or as an effect of the response. ${ }^{1}$ The letters D and F were mapped to one key and the letters J and $\mathrm{K}$ to the other key. Following this first response, in most of the trials a second (probe) sound was presented individually. Participants were instructed to press either key in response to this stimulus. They were asked to select this response key randomly but try to use both keys for about the same number of trials throughout the experiment.

In $20 \%$ of the trials, no probe sound was presented and participants had to refrain from a second response. These trials were included to minimize response strategies that included rhythmic responding. In sound repetition trials (i.e., distractor repetition and effect repetition trials), the previous distractor sound or effect sound was repeated as the probe sound. In sound change trials, the probe sound differed from the sound

\footnotetext{
${ }^{1}$ Note that objectively simultaneously presented visual and auditory stimuli might subjectively slightly diverge in time (Rutschmann \& Link, 1964). We thank an anonymous reviewer for this remark.
} 
Fig. 1 a Trial procedure in Experiment 1 (stimuli not drawn to scale). On the prime, participants responded to the identity of a target letter by pressing the according key. An additional, task-irrelevant sound either accompanied the prime target (distractor blocks; upper part) or followed as an effect of the prime response (effect blocks; lower part). On the probe, participants responded by pressing randomly one of the response keys as soon as the probe sound was presented. $\mathbf{b}$ Binding effects (in percentages) for distractor blocks and effect blocks of Experiment 1, calculated as the difference between response repetition rates of sound repetition and sound change trials, with positive values indicating prime-consistent responding. Error bars depict standard errors of paired differences, computed separately for each response relation (Pfister \& Janczyk, 2013). c Distractorresponse (D-R) binding and response-effect (R-E) binding effects in Experiment 1; points represent individual participants a

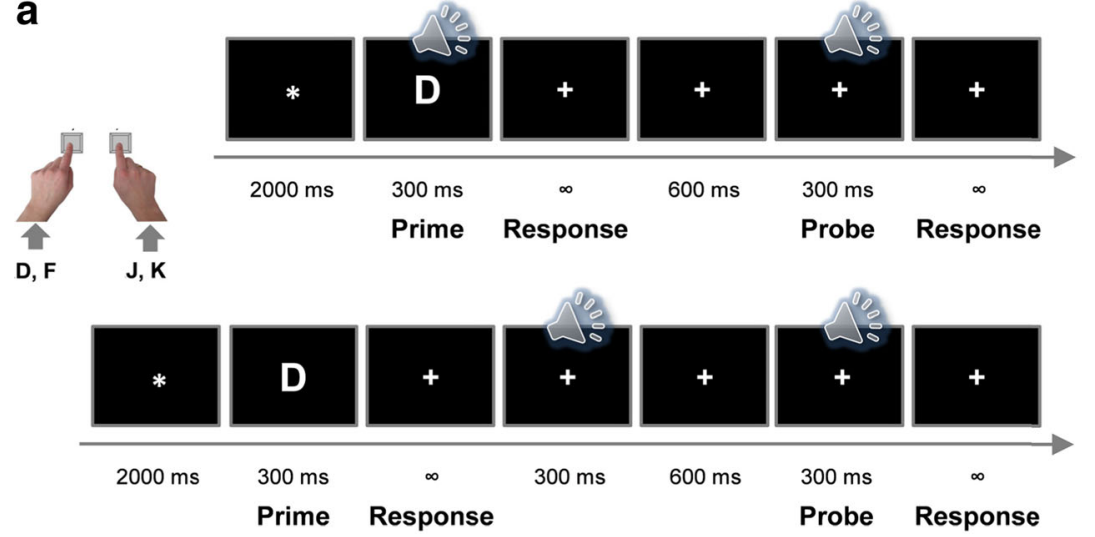

b

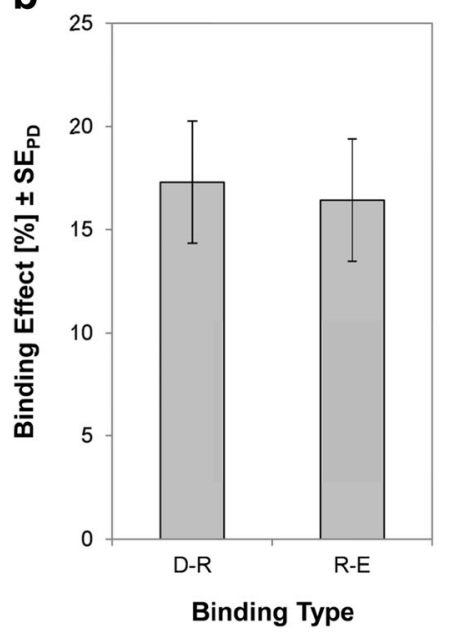

C

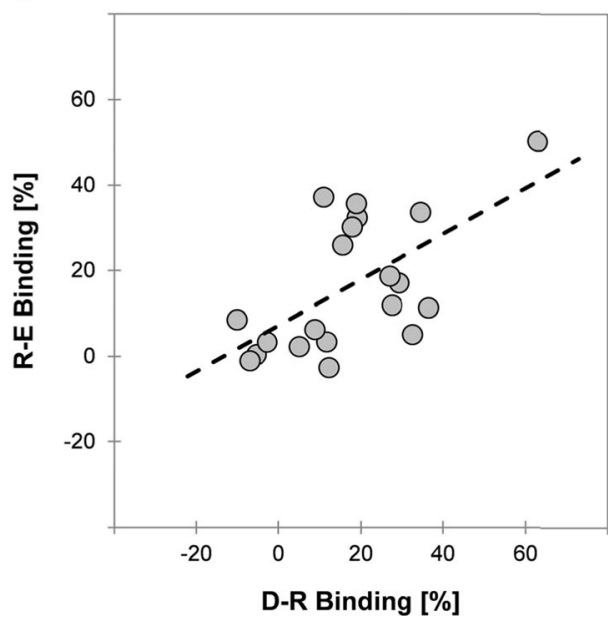

that had been presented during the prime. Importantly, in this and all following experiments, there were no contingencies between sound identities and required responses.

Each participant worked through one experimental block including prime distractor stimuli and one block including prime response effects (Fig. 1a). In effect blocks, each trial started with $2000 \mathrm{~ms}$ presentation of an asterisk. Then, the prime target appeared for $300 \mathrm{~ms}$. Each prime response triggered a $300-\mathrm{ms}$ effect sound; $600 \mathrm{~ms}$ after this effect, the probe sound appeared for $300 \mathrm{~ms}$ and participants responded by pressing either of the response keys. Distractor blocks were identical to effect blocks except that a distractor sound now accompanied each prime target and responses did not produce any auditory effects.

Each block comprised 200 prime-probe sequences and block order was counterbalanced across participants. Repetition and change of the additional stimulus was realized in 80 trials each, both for the prime distractors and prime response effects. Forty catch trials were included in each block. Before each experimental block, participants worked through a practice block of 20 prime-probe sequences in which they received feedback after each response. Everything else was as in the experimental blocks. To familiarize participants with the stimulus material, they were given an additional set of 20 practice trials at the beginning of the experiment.

\section{Results}

Only correct trials were considered for analyses of the choice data. We further excluded all catch trials and post-error trials. Due to these constraints, $15.1 \%$ of all trials were discarded (prime error rate for distractor blocks was $7.6 \%$, and prime error rate for effect blocks was $5.3 \%$ ). Overall, participants showed response repetitions in about half of the trials, both in distractor blocks (47.5\% response repetitions) and in effect blocks (50.8\% response repetitions). These response repetition rates were not significantly different from chance, $t(19)=$ $-0.68, p=0.504, d=0.15$, for distractor relation and $t(19)=$ $0.22, p=0.829, d=0.05$, for effect relation, respectively. False alarms in the catch trials were infrequent for both, distractor blocks ( $M=1.1 \%$ of all catch trials, $S D=1.9 \%)$ and effect blocks $(M=5.6 \%, S D=4.9 \%)$.

Our dependent variable of interest was the rate with which participants repeated their response from prime to probe. As 
expected, we observed more response repetitions in effect blocks when the probe sound resembled the previous effect $(58.8 \%)$ than when the sounds changed from prime to probe (42.4\%), $t(19)=4.72, p<0.001, d=1.06$ (see Fig. $1 b$; d was computed as $\mathrm{t} / \mathrm{sqrt}(\mathrm{n}))$. Importantly, this also was true for distractor blocks, with more response repetitions when the probe sound resembled the previous distractor $(56.1 \%)$ than when the sounds changed (38.8 \%), $t(19)=4.39, p<0.001, d=0.98$.

To further compare the two binding effects, we ran a 2 (sound relation: repetition vs. change) $\times 2$ (role of the sounds: distractor vs. effect) repeated measures analysis of variance (ANOVA) on the response repetition rates. This analysis confirmed the above tests of the individual effects by means of a significant main effect of sound relation, $F(1,19)=25.66, p<$ $0.001, \eta_{\mathrm{p}}{ }^{2}=0.58$. Importantly, the interaction of sound relation and role of the sounds was far from significant, $F<1$, indicating no difference between binding for distractor stimuli and response effects. Mean repetition rates did not differ between blocks, $F<1$.

For follow-up correlation analysis of the response repetition data, we computed sound repetition effects on the relative frequencies $(f)$ of response repetitions $-f$ (response repetition | sound repetition) - $f$ (response repetition $\mid$ sound change) - for each block type and participant (Fig. 1c). The two sound repetition effects were indeed correlated across participants, $r=$ $0.608, p=0.004$, indicating interindividual differences to vary systematically across both binding effects.

The same analysis of the probe RTs also revealed binding effects that were not modulated by the role of the additional sound. In a 2 (sound role in the prime: distractor vs. effect) $\times 2$ (response relation: repetition vs. change) $\times 2$ (sound relation: repetition vs. change) repeated measures ANOVA, the main effect of response relation was significant $F(1,19)=8.23, p=$ $0.010, \eta_{\mathrm{p}}{ }^{2}=0.30$, indicating faster responses if participants changed the response side from prime to probe $(M=390 \mathrm{~ms}$, $S D=115 \mathrm{~ms})$ than if they repeated the response side $(M=$ $407 \mathrm{~ms}, S D=97 \mathrm{~ms}$ ). Responses tended to be faster in effect blocks than in distractor blocks, $F(1,19)=4.14, p=0.056, \eta_{\mathrm{p}}{ }^{2}$ $=0.18$. More interestingly, the interaction of response relation and sound relation was significant, $F(1,19)=8.96, p=0.007$, $\eta_{\mathrm{p}}{ }^{2}=0.32$, indicating binding between the prime response and the sound. This interaction effect, however, was not further qualified by the role of the sound (distractor vs. effect), $F(1$, 19) $=0.28, p=0.603, \eta_{\mathrm{p}}{ }^{2}=0.02$, suggesting that the type of binding did not influence the size of binding effects (see Table 1 for the mean probe RTs). None of the other effects was significant, $F(1,19)<3.1 ; p>0.095 ; \eta_{\mathrm{p}}{ }^{2}<0.14$.

\section{Discussion}

The results of Experiment 1 clearly indicated that both bindings between distractor-stimuli and responses as well as between responses and effects can be measured in an experimental paradigm that was initially designed to probe for responseeffect binding (Dutzi \& Hommel, 2009; Herwig \& Waszak, 2012; Janczyk et al., 2012). Furthermore, the two binding effects did not differ from each other and were correlated across participants. To validate these findings, we opted for the complementary approach and probed for response-effect binding in a design that is typically used for investigating distractor-response binding.

\section{Experiment 2}

\section{Method}

Participants Twenty-five students (19 females) from the University of Trier took part in the experiment. Their median age was 22 (range 18-32) years. Four participants had to be replaced because of extremely slow RTs (mean RT more than 1.5 interquartile ranges above the third quartile of the remaining sample in both blocks) or an extreme percentage of errors in either the distractor or the effect block. Participants received course credit or monetary compensation.

Materials and procedure Materials and methods were the same as in Experiment 1, except for the probe procedure (Fig. 2a). Participants now responded to a target letter both on the prime and on the probe, and the probe target either required
Table 1 Mean reaction times (in $\mathrm{ms}$ ) in Experiment 1 as a function of sound relation and role of the sound during the prime

\begin{tabular}{lll}
\hline & \multicolumn{2}{l}{ Response relation } \\
\cline { 2 - 3 } & Response repetition (RR) & Response change (RC) \\
\hline Prime distractors & & \\
Distractor change (DC) & 451 & 401 \\
Distractor repetition (DR) & 411 & 401 \\
Prime effects & & 372 \\
Effect change (EC) & 403 & 385 \\
Effect repetition (ER) & 385 &
\end{tabular}


Fig. 2 a Trial procedure in Experiment 2 (stimuli not drawn to scale). Participants responded to the identity of a target letter both, on the prime and on the probe. An additional task irrelevant sound was presented accompanying the prime target (distractor blocks; upper part) or as an effect of the prime response (effect blocks; lower part). The probe target was always accompanied by a task irrelevant sound. b Binding effects (in ms) for distractor blocks and effect blocks Experiment 2, calculated as the difference between sound repetition effects in response repetition and response change trials. Error bars depict standard errors of paired differences, computed separately for each response relation (Pfister \& Janczyk, 2013). c Distractorresponse (D-R) binding and response-effect (R-E) binding effects in Experiment 2; points represent individual participants

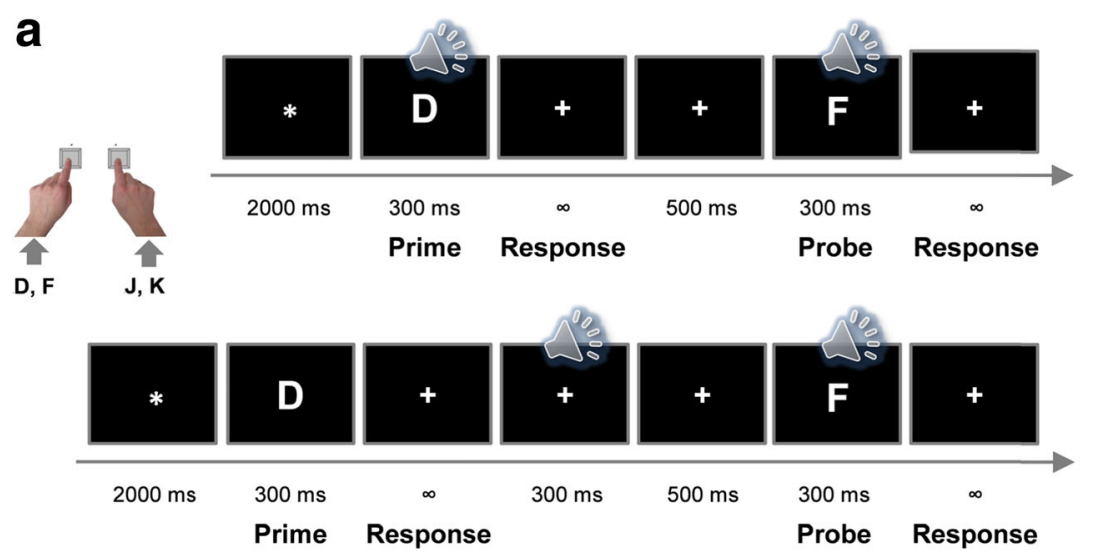

b

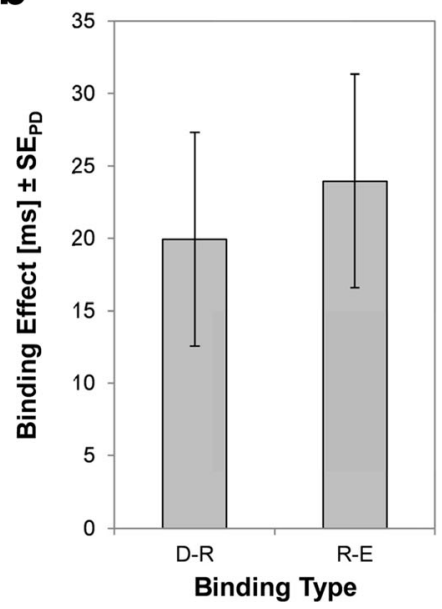

C

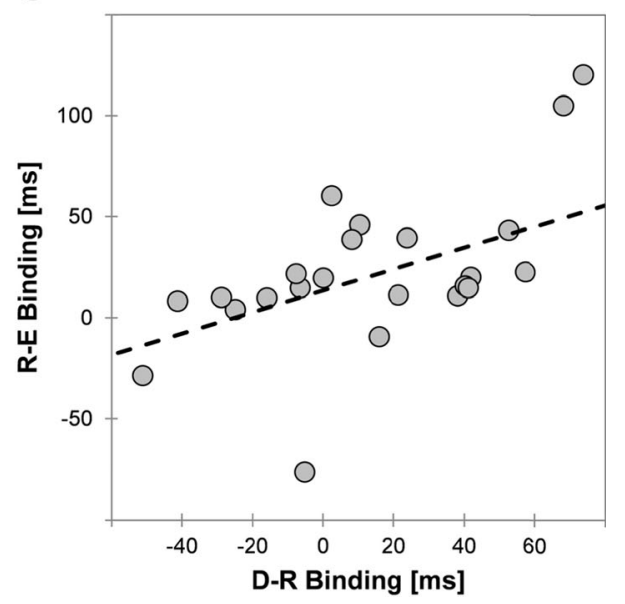

the same response as the prime target or the alternative response and it was always accompanied by an additional sound. In contrast to Experiment 1, there were no catch trials, conforming to typical procedures in research on distractor-response binding (Frings et al., 2007; Rothermund et al., 2005).

Sound repetition from prime to probe and role of the sound during the prime (distractor vs. effect) were varied orthogonally to response relation. In response repetition trials, the probe target required the same response as the prime target. In response change trials different responses were required on the prime and the probe. In sound repetition trials, the same task irrelevant sound that had been presented on the prime was again presented together with the probe target. On sound change trials, the sound presented during the probe differed from the sound presented on the prime.

The order of effect and distractor blocks was randomized across participants. Each of these experimental blocks comprised 192 trials. The four conditions response repetition / sound repetition, response repetition / sound change, response change / sound repetition, and response change / sound change were each realized in 48 trials, both for distractor and effect blocks. Participants practiced their task for 32 trials at the beginning of the experiment and for 8 trials before the second experimental block started. During practice, they received feedback after each response. Other than that, the task was exactly as during the experimental blocks.

\section{Results}

Only correct trials were considered for the analyses and we again excluded post-error trials. RTs that were more than 1.5 interquartile ranges above the third quartile of the RT distribution of the participant (Tukey, 1977), and those that were shorter than $200 \mathrm{~ms}$ were excluded from the analysis $(4.21 \%)$. Due to these constraints, $18.3 \%$ of all trials were discarded (probe error rate of all trials with correct prime responses was $4.5 \%$, prime error rate of all trials was $3.6 \%$ ). Furthermore, trials with target repetitions were excluded from the analysis. Repeating this analysis including target repetition trials revealed the same pattern. Mean RTs and error rates for analyzed probe displays are depicted in Table 2.

Probe RTs of the distractor blocks were analyzed by means of a 2 (response relation: repetition vs. change) $\times 2$ (sound relation: repetition vs. change) repeated measures ANOVA. As expected, we observed distractor-response binding in terms of a significant interaction of response relation and 
Table 2 Mean reaction times (in $\mathrm{ms}$ ) and mean error rates (in percentage) in Experiment 2 as a function of sound relation and role of the sound during the prime
Response relation

Response repetition (RR)

Response change (RC)

$\begin{array}{lll}\text { Prime distractors } & \\ \text { Distractor change (DC) } & 547(8.1) & 533(2.7) \\ \text { Distractor repetition (DR) } & 547(7.2) & 552(6.2) \\ \text { Prime effects } & & \\ \text { Effect change (EC) } & 539(9.6) & 523(4.3) \\ \text { Effect repetition (ER) } & 534(5.7) & 543(6.7)\end{array}$

sound relation, $F(1,24)=6.38, p=0.019, \eta_{\mathrm{p}}{ }^{2}=0.21$. Furthermore, the main effect of sound repetition was significant, $F(1,24)=10.87, p=0.003, \eta_{\mathrm{p}}{ }^{2}=0.31$, indicating longer RTs if the sound repeated than if it changed, whereas the main effect of response relation was not significant, $F<1$. Notably, we found an identical pattern in the analysis of the effect blocks with a significant interaction of response relation and sound relation, $F(1,24)=9.85, p=0.004, \eta_{\mathrm{p}}{ }^{2}=0.29$, indicating short-term response-effect bindings. The main effect of sound repetition was significant, $F(1,24)=11.40, p=0.002$, $\eta_{\mathrm{p}}{ }^{2}=0.32$, whereas the main effect of response relation was not significant, $F<1$.

In a follow-up analysis, we again compared distractorresponse and response-effect binding. This 2 (sound role in the prime: distractor vs. effect) $\times 2$ (response relation: repetition vs. change) $\times 2$ (sound relation: repetition vs. change) repeated measures ANOVA on probe RTs did not yield a three-way interaction of sound role, response relation, and sound relation, $F<1$, indicating no difference between binding for distractor stimuli and response effects (see Fig. $2 \mathrm{~b}$ for a comparison of both binding effects, computed as $\left(\mathrm{RT}_{\text {sound }}\right.$ change $-\mathrm{RT}_{\text {sound }}$ repetition $)_{\text {response repetition }}-\left(\mathrm{RT}_{\text {sound }}\right.$ change $\mathrm{RT}_{\text {sound repetition) }}$ response change).

In a final analysis of the probe RT data, we addressed the correlation of distractor-response bindings and response-effect bindings across participants (computed as described above). As in Experiment 1, the two binding effects were positively correlated, $r=.549, p=.005$, again indicating interindividual differences to vary together in both binding effects (Fig. 2c). ${ }^{2}$

The same analyses on error rates showed a similar pattern. The interaction of response relation and sound relation was significant, both for distractor blocks, $F(1,24)=5.93, p=0.023, \eta_{\mathrm{p}}{ }^{2}$ $=0.20$, and effect blocks, $F(1,24)=9.85, p=0.004, \eta_{\mathrm{p}}{ }^{2}=0.29$, and this binding effect was not modulated by stimulus role, $F<$

\footnotetext{
${ }^{2}$ Split-half reliabilities for both effects were lower than the reported correlations. This might be due to the smaller number of trials used for this analysis. In addition, reliabilities of RT-based tasks like Negative Priming, Stroop or the Flanker task typically produce low to medium reliabilities (e.g., Friedman \& Miyake, 2004). Yet, in concert with the data of Experiment 1, in which split-half reliabilities approached satisfying sizes and were significant, we argue that this analysis supports the notion of a common binding mechanism.
}

1. However, the correlation of binding effects in error rates did not reach significance, $r=0.149, p=0.478$.

\section{Discussion}

The results of Experiment 2 indicate that response-effect binding can influence responding in forced choice RT tasks if a stimulus that was previously triggered as an action-contingent effect is presented simultaneously with the target. Notably, bindings between responses and effects were again strongly correlated with distractor-response bindings. Together with the findings of Experiment 1, this can be taken as a first indication that integration of responses with any kind of stimuli might be mediated by the same mechanism, irrespective of whether these stimuli assume the roles of task-irrelevant distractors or self-produced effects.

\section{Experiment 3}

A final implication of the assumption that distractor-response and response-effect bindings rely on the same mechanism is that both effects should be similarly modulated by factors that have previously been shown to affect one binding effect but have not yet been studied for the other. For instance, bindings between distractor stimuli and responses are known to decay rapidly over time, leading to significantly smaller effects for long $(2000 \mathrm{~ms})$ than for short $(500 \mathrm{~ms})$ response-stimulus intervals (Frings, 2011). There is currently no data to suggest a similar impact of effect-stimulus interval for response-effect bindings. Assuming a shared mechanism underlying both effects, however, longer intervals between effect and probe stimulus presentation should likewise yield weaker evidence for response-effect bindings. Following this reasoning, we conducted a third experiment that varied intervals between binding during the prime (i.e., the time of the prime response, or time of effect presentation, respectively) and retrieval (i.e., onset of the probe). We expected an impact of retrieval delay for distractor-response binding, replicating previous findings (Frings, 2011), and crucially also for response-effect bindings. 


\section{Method}

Participants Thirty-one students (25 females) from the University of Trier took part in the experiment. Their median age was 22 (range 19-26) years. One participant had to be replaced because of extremely slow RTs (mean RT more than 1.5 interquartile ranges above the third quartile of the remaining sample for both retrieval delay conditions). Participants received course credit or monetary compensation.

Materials and procedure Materials and methods were the same as in Experiment 2, with the following exceptions. Each participant worked through two blocks including prime distractor sounds and through two blocks including prime response effects. For both conditions, one of these blocks included $500 \mathrm{~ms}$ intervals between binding in the prime (i.e., time of responding in distractor and effect onset in effect blocks, respectively) and probe onset and therefore was identical to the respective block in Experiment 2. The other block included longer intervals of $2000 \mathrm{~ms}$ between binding in the prime and onset of the probe. Participants randomly either started with the distractor sound or with the sound effect blocks. The order of retrieval delays (500 ms vs. $2000 \mathrm{~ms}$ ) was identical for both block types but randomized across participants. Each block included 192 trials. Before the first block of either the distractor or the effect sound condition started, participants practiced responding in the new setup for 16 trials. During practice, they received feedback after every response. Other than that the procedure was identical to the experimental blocks.

\section{Results}

Due to the same constraints as in Experiment 2, 23.4\% of all trials were discarded (probe error rate of all trials with correct prime responses was $6.0 \%$, prime error rate of all trials was $4.4 \% ; 5.21 \%$ RT outliers). As in Experiment 2, trials with target repetitions were excluded from the analyses. Mean RTs and error rates for analyzed probe displays are depicted in Table 3.

Probe RTs were analyzed by means of a 2 (retrieval delay: $500 \mathrm{~ms}$ vs. $2000 \mathrm{~ms}$ ) $\times 2$ (sound role in the prime: distractor vs. effect) $\times 2$ (response relation: repetition vs. change) $\times 2$ (sound relation: repetition vs. change) repeated measures ANOVA. The main effects of retrieval delay, $F(1,30)=$ $35.65, p<0.001, \eta_{\mathrm{p}}{ }^{2}=0.54$, and sound role in the prime, $F(1,30)=8.73, p=0.006, \eta_{\mathrm{p}}{ }^{2}=0.23$, were significant, indicating faster responses with long retrieval delays than with short ones, and faster responses in distractor blocks than in effect blocks. We further observed a general binding effect in terms of a significant interaction of response relation and sound relation, $F(1,30)=36.94, p<0.001, \eta_{\mathrm{p}}{ }^{2}=0.55$. Most importantly, binding was significantly modulated by retrieval delay (three-way interaction of response relation $\times$ sound relation $\times$ retrieval delay), $F(1,30)=10.75, p=0.003, \eta_{\mathrm{p}}{ }^{2}=$ 0.264 (Fig. 3). Notably, neither the general binding effect, nor the binding $\times$ retrieval delay interaction were further modulated by sound role in the prime (response relation $\times$ sound relation $\times$ sound role in the prime: $F(1,30)=1.37, p=$ $0.252, \eta_{\mathrm{p}}{ }^{2}=0.04$; and retrieval delay $\times$ response relation $\times$ sound relation $\times$ sound role in the prime: $F<1, \eta_{\mathrm{p}}{ }^{2}=0.01$ ). Additional analyses indicated that the binding effect was larger in trials with 500-ms retrieval delay than in 2000-ms retrieval delay both for distractor sounds, $F(1,30)=4.27, p=$ $0.047, \eta_{\mathrm{p}}{ }^{2}=0.13$, and also for sound effects, $F(1,30)=6.41, p$ $=0.017, \eta_{\mathrm{p}}{ }^{2}=0.176$. None of the other effects reached significance, all $F(1,30)<2.7, p>0.111, \eta_{\mathrm{p}}{ }^{2}<0.09 .^{3}$

The error rates showed a similar pattern. The main effects for retrieval delay, $F(1,30)=7.70, p=0.009, \eta_{\mathrm{p}}{ }^{2}=0.20$, and response relation, $F(1,30)=12.75, p=0.001, \eta_{\mathrm{p}}{ }^{2}=0.30$, were significant. The interaction of response relation and sound relation was significant, as well, $F(1,30)=20.36, p<0.001$, $\eta_{\mathrm{p}}{ }^{2}=0.40$, and tended to be modulated by retrieval delay, $F(1$, $30)=3.43, p=0.074, \eta_{\mathrm{p}}{ }^{2}=0.10$, whereas sound role in the prime did not modulate the response relation $\times$ sound relation interaction $\left(F<1, \eta_{\mathrm{p}}{ }^{2}<0.01\right)$ or the retrieval delay $\times$ response relation $\times$ sound relation interaction $\left(F<1, \eta_{\mathrm{p}}{ }^{2}<0.02\right)$. None of the other effects was significant.

Although we were mainly interested in bindings between responses and additional stimuli, it should be noted that bindings between targets and distractors can influence performance independently of bindings between distractors and responses (Giesen \& Rothermund, 2014). Therefore, in a final analysis, we took also target repetition trials into account, which further differentiated the result pattern. In a 3 (Response Relation: target repetition vs. response repetition vs. response change) $\times 2$ (Sound Relation: repetition vs. change) $\times 2$ (Sound Role: distractor vs. effect) $\times 2$ (RSI: $500 \mathrm{~ms}$ vs. $2000 \mathrm{~ms})$ ANOVA on probe RTs, contrasts indicated a different pattern for target integration than for response integration. While binding between response and sound was similar for distractor and effect sounds, $F(1,30)=1.37, p=0.252, \eta_{\mathrm{p}}{ }^{2}=0.04$, binding between sound and target was significantly modulated by sound role, $F(1,30)=7.24, p=0.012, \eta_{\mathrm{p}}{ }^{2}=0.19$ : Targets were more strongly integrated with distractor sounds than with sound effects. In addition, variation in response stimulus interval modulated binding between sounds and responses, $F(1,30)=10.75, p=$ $0.003, \eta_{\mathrm{p}}{ }^{2}=0.26$, but not between sounds and targets, $F(1,30)=$

\footnotetext{
${ }^{3}$ The correlation between both binding effects was not significant in this experiment $(r=-0.133, p=0.476)$. This observation deviates from the result pattern predicted by a common underlying mechanism. Note however, that Experiment 3 was designed with the intention to compare mean RTs. Therefore, sequences of the four blocks were counterbalanced between participants. As a side effect, correlations were affected to a much larger extent by interactions of the interindividual variance and sequence effects in Experiment 3 as compared to the first two experiments, yielding increased possibility for Type II errors.
} 
Table 3 Mean reaction times (in $\mathrm{ms}$ ) and mean error rates (in percentage) in Experiment 3 as a function of sound relation, retrieval delay, and role of the sound during the prime

\begin{tabular}{|c|c|c|c|c|}
\hline & \multicolumn{2}{|c|}{ Retrieval delay $500 \mathrm{~ms}$} & \multicolumn{2}{|c|}{ Retrieval delay $2000 \mathrm{~ms}$} \\
\hline & $\begin{array}{l}\text { Response repetition } \\
\text { (RR) }\end{array}$ & $\begin{array}{l}\text { Response change } \\
\text { (RC) }\end{array}$ & $\begin{array}{l}\text { Response repetition } \\
\text { (RR) }\end{array}$ & $\begin{array}{l}\text { Response change } \\
\text { (RC) }\end{array}$ \\
\hline \multicolumn{5}{|l|}{ Prime distractors } \\
\hline $\begin{array}{l}\text { Distractor change } \\
\text { (DC) }\end{array}$ & $528(13.4)$ & $511(4.8)$ & $555(7.9)$ & $539(4.5)$ \\
\hline $\begin{array}{l}\text { Distractor } \\
\text { repetition (DR) }\end{array}$ & $519(8.4)$ & $535(8.2)$ & $547(7.4)$ & $548(7.0)$ \\
\hline \multicolumn{5}{|l|}{ Prime effects } \\
\hline Effect change (EC) & $505(12.4)$ & $497(4.5)$ & $534(9.3)$ & $531(4.1)$ \\
\hline $\begin{array}{l}\text { Effect repetition } \\
\text { (ER) }\end{array}$ & $492(8.6)$ & $512(8.8)$ & $536(8.3)$ & $536(8.2)$ \\
\hline
\end{tabular}

$1.21, p=0.280, \eta_{\mathrm{p}}{ }^{2}=0.04$. That is, although there was a clear difference in sound processing for distractors and effects, integration of the additional stimulus with the current response was not affected by it. ${ }^{4}$

\section{Discussion}

The results of Experiment 3 indicate that varying the length of the interval between binding and retrieval of stimulusresponse associations has a similar effect on distractorresponse and on response-effect bindings. We observed significantly larger binding effects for short than for long retrieval delays, and this modulation did not differ for distractorresponse and for response-effect bindings. This was the case, although including target repetition trials in the analysis indicated response-unrelated differences in distractor and effect stimulus processing. Thus, Experiment 3 provides further indication that binding of distractors and responses and binding of responses and effects covary with the same experimental manipulation, which further supports the idea that they are brought about by the same general mechanism.

\section{General discussion}

The present study targeted the mechanisms underlying the incidental acquisition of short-term associations between responses and concurrent or closely following stimuli. In the first two of three experiments, we compared distractorresponse binding on the one hand and response-effect binding on the other hand by measuring each effect in its native experimental design and in a non-native analogue that is typically used for investigating the alternative effect. Both effects

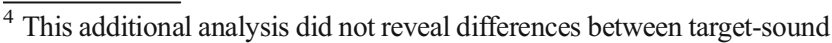
integrations for distractor and effect sounds in Experiment 2. Yet, the results of Experiment 3 indicate that even in situations in which distractor and effect sounds are differently processed with regard to their target relation, integration with a response can be identical.
}

occurred consistently in both designs, suggesting that distractor-response and response-effect bindings might be driven by the same underlying processes. In addition, the magnitudes of both binding effects were similar in both experiments, indicating that neither the experimental design nor any inherent characteristics of either effect biased their emergence. Moreover, in a third experiment, we manipulated a potential modulating factor for both binding effects and found both to be similarly affected by retrieval delay.

Our results thus provide evidence for the assumption that binding is a widely unselective and automatic process that follows a rule of thumb to bind anything available in an integration window that stretches (at least) from the time of responding to closely following stimulation. This conclusion

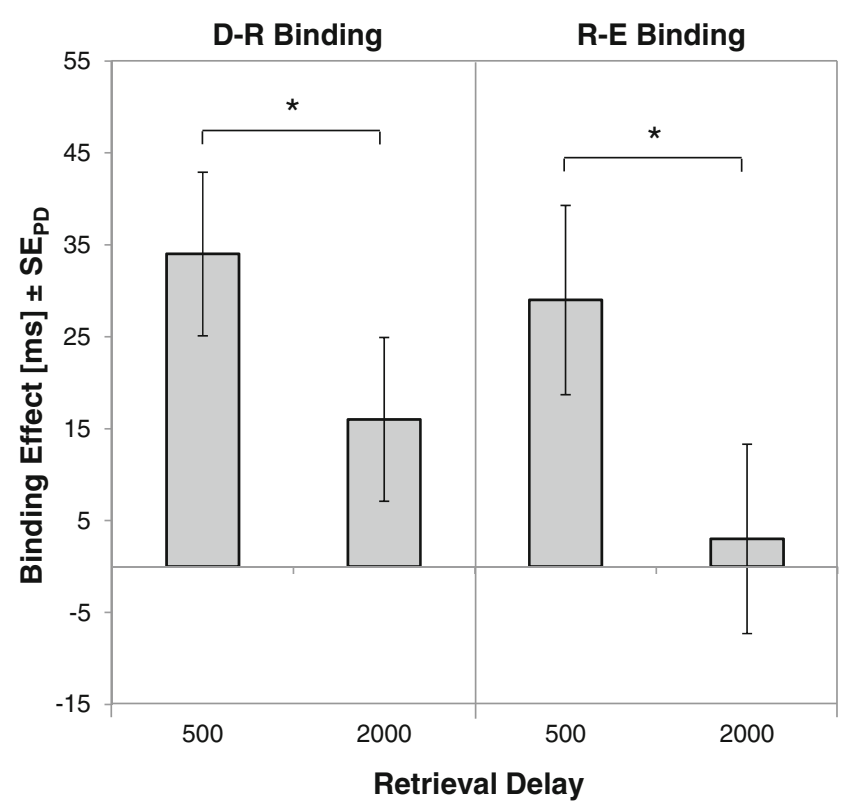

Fig. 3 Binding effects (in ms) for distractor blocks and effect blocks in Experiment 3, depicted as a function of retrieval delay $(500 \mathrm{~ms}$ vs. $2000 \mathrm{~ms}$ ). Binding is calculated as the difference between sound repetition effects in response repetition and response change trials. Error bars depict standard errors of paired differences (Pfister \& Janczyk, 2013) 
is further supported by a consistent and sizeable correlation between distractor-response binding and response-effect bindings in the first two experiments. We take this as evidence that the strength of the associative mechanism is rather consistent within each individual. Note that this correlation is by no means a necessary consequence if the two effects can be explained by a single mechanism such as event-file binding (Dutzi \& Hommel, 2009; Frings et al, 2007; Hommel, 2004). For instance, compatibility effects that emerge either from dimensional overlap of stimuli and responses (S-R compatibility; Kornblum, Hasbroucq, \& Osman, 1990; Proctor \& Reeve, 1990) or from dimensional overlap of responses and following effects (R-E compatibility; Kunde, 2001; Pfister \& Kunde, 2013) can likewise be accommodated in a single theoretical framework but were found not to be correlated across individuals (Keller \& Koch, 2006).

It also is important to note that the similarity of the two effects and the similar modulation due to a third factor cannot be taken as unambiguous proof of a common underlying mechanism. A large body of evidence, failing to find differences between the processes, would be necessary to further validate this point. With the present study we take an important first step in this process. At this point, it is worth noting that the distractor-response and response-effect binding effects not only correlated in Experiment 1 and 2 but that they behaved in very similar ways in three different experiments, even though response-unrelated distractor and effect processing are not necessarily identical (see Experiment 3). That is, the combined result pattern of the three experiments clearly favors an explanation in terms of a common mechanism.

The possibility that both effects are driven by the same process is all the more surprising, considering the inherent differences between distractors and effects regarding their temporal proximity to the response. Distractor stimuli are obviously present at the time of response selection and execution, whereas effects follow by definition only after a response has been initiated. That is, distractors are present at the time of event file formation, while effects have to be integrated in an already existing event file. Assuming a decaying representation of the event file over time, in some cases effects may even have to be integrated into already decaying event files. One might therefore expect decreased binding with decreasing temporal contiguity of response and effect (Elsner \& Hommel, 2004). With the effect following immediately after response execution and effect duration of $300 \mathrm{~ms}$, it seems likely that the current effects still fell within a time window well before decay onset (Akyürek \& Hommel, 2005; Frings, 2011; Herwig \& Waszak, 2012). The exact size of such an integration window is still a matter of debate (Akyürek, Toffanin, \& Hommel, 2008; Dignath, Pfister, Eder, Kiesel \& Kunde, 2014; Humphreys \& Buehner, 2009), but the existence of such a window seems to ensure strong bindings at least for those events that follow promptly after a response.
Given the present finding that distractor-response and response-effect bindings seem to be driven by the same mechanism, it can be speculated that grouping of to be integrated stimuli and/or responses plays a role for the definition of an integration window. Distractor-response binding has indeed been shown to increase with target-distractor grouping (Frings \& Rothermund, 2011; Giesen \& Rothermund, 2011; Moeller, Rothermund, \& Frings, 2012) and such grouping can apparently be mediated by temporary differences in target and distractor appearance (Frings \& Moeller, 2012). Therefore, it seems possible that depending on temporal grouping conditions, integration windows for response-effect binding may vary (Akyürek, Toffanin, \& Hommel, 2008).

At first sight, our results seem to disagree with former studies reporting a prevalence of response-effect association formation over stimulus-response association formation (Hoffmann \& Sebald, 2000; Stock \& Hoffmann, 2002), because we did not observe any differences between binding of effects and binding of additional distractor stimuli. Yet, it should be noted that these earlier studies investigated associations at a different timeframe and also differed with regard to the participants' intentions. While Hoffmann and colleagues were concerned with learning long-term associations through repeated experience, we analyzed immediate binding effects after a single response/stimulus encounter. Hence, our findings may be an indication that the earliest processes in association learning are entirely automatic and do not discriminate between the roles of integrated stimuli. According to this interpretation, differences between the integration of different stimuli with a current response only occur later in the process (Herwig \& Waszak, 2012), possibly due to attentional distribution when certain stimuli occur repeatedly. In addition, it is also possible that differences between long-term stimulus and effect-integration arise from the intention to produce a certain effect (Hoffmann \& Sebald, 2000). Participants in the present study were never instructed to produce certain effects. With a random response-effect assignment in each trial and the fact that even in the effect-blocks only half of the participants' responses led to effects (i.e., only prime responses), it is unlikely that participants redefined their task as to producing effects. In contrast, participants in the mentioned studies reporting differences between stimulus-response and response-effect learning always intended to produce certain effects (Herwig \& Waszak, 2009; Moeller \& Frings, 2014b for the impact of attention on distractor-response binding).

Finally, the results of Experiment 1 demonstrate for the first time that the selection of a freely chosen response can be influenced by bindings between responses and distractors. That is, a formerly ignored stimulus that has been associated with a response does not only modulate the readiness with which an action is executed, but can even influence the decision for this action. Therefore, the present finding points 
toward an entire new range of situations in which action control is influenced by distractor-response bindings.

In conclusion, the present study indicates that incidental binding of task-irrelevant stimuli and responses is likely due to a very robust and rather non-selective process. Particularly, distractor-response and response-effect bindings seem to be driven by the same mechanism; this mechanism of forming short-term associations between own responses and any kind of surrounding stimuli seems to be sufficient to exploit a wide variety of environmental contingencies for action control.

Acknowledgments The research reported in this article was supported by grants of the Deutsche Forschungsgemeinschaft to Christian Frings (FR 2133/1-2) and Birte Moeller (MO 2839/2-1).

\section{References}

Akyürek, E. G., \& Hommel, B. (2005). Target integration and the attentional blink. Acta Psychologica, 119, 305-314.

Akyürek, E. G., Toffanin, P., \& Hommel, B. (2008). Adaptive control of event integration. Journal of Experimental Psychology: Human Perception and Performance, 34, 569-577.

Botvinick, M. (2007). Conflict monitoring and decision making: Reconciling two perspectives on anterior cingulate function. Cognitve, Affective, \& Behavioral Neuroscience, 7, 356-366.

Cardoso-Leite, P., Mamassian, P., Schütz-Bosbach, S., \& Waszak, F. (2010). A new look at sensory attenuation action-effect anticipation affects sensitivity, not response bias. Psychological Science, 21, 1740-1745.

Cohen, J., McClure, S., \& Yu, A. (2007). Should I stay or should I go? How the human brain manages the trade-off between exploitation and exploration. Philosophical Transactions of the Royal Society B, 362, 933-942.

Dignath, D., Pfister, R., Eder, A. B., Kiesel, A., \& Kunde, W. (2014). Representing the hyphen in action-effect associations: Automatic acquisition and bidirectional retrieval of action-effect intervals. Journal of Experimental Psychology: Learning, Memory, and Cognition, 40, 1701-1712.

Dutzi, I., \& Hommel, B. (2009). The microgenesis of action-effect binding. Psychological Research, 73, 425-435.

Elsner, B., \& Hommel, B. (2001). Effect anticipation and action control. Journal of Experimental Psychology: Human Perception and Performance, 27, 229-240.

Elsner, B., \& Hommel, B. (2004). Contiguity and contingency in the acquisition of action effects. Psychological Research, 68, 138-154.

Friedman, N., \& Miyake, A. (2004). The relations among inhibition and interference control functions: A latent-variable analysis. Journal of Experimental Psychology: General, 133, 101-135.

Frings, C. (2011). On the decay of distractor-response episodes. Experimental Psychology, 58, 125-131.

Frings, C., \& Moeller, B. (2010). Binding targets' responses to distractors' locations: Distractor response bindings in a location priming task. Attention, Perception, \& Psychophysics, 72, 21762183.

Frings, C., \& Moeller, B. (2012). The horserace between distractors and targets: Retrieval-based probe responding depends on distractortarget asynchrony. Journal of Cognitive Psychology, 24, 582-590.

Frings, C., Moeller, B., \& Rothermund, K. (2013). Retrieval of event files can be conceptually mediated. Attention, Perception, \& Psychophysics, 75, 700-709.
Frings, C., \& Rothermund, K. (2011). To be, or not to be...included in an event file: When are distractors integrated into S-R episodes and used for response retrieval? Journal of Experimental Psychology: Learning, Memory, and Cognition, 37, 1209-1227.

Frings, C., Rothermund, K., \& Wentura, D. (2007). Distractor repetitions retrieve previous responses to targets. Quarterly Journal of Experimental Psychology, 60, 1367-1377.

Garner, W. R., \& Felfoldy, G. L. (1970). Integrality of stimulus dimensions in various types of information processing. Cognitive Psychology, 1, 225-241.

Giesen, C., \& Rothermund, K. (2011). Affective matching moderates S-R binding. Cognition \& Emotion, 25, 342-350.

Giesen, C., \& Rothermund, K. (2014). Distractor repetitions retrieve previous responses and previous targets: Experimental dissociations of distractor-response and distractor-target bindings. Journal of Experimental Psychology: Learning, Memory, and Cognition, 40(3), 645-659.

Haggard, P., \& Tsakiris, M. (2009). The experience of agency: Feelings, judgments, and responsibility. Current Directions in Psychological Science, 18, 242-246.

Henson, R. N., Eckstein, D., Waszak, F., Frings, C., \& Horner, A. J. (2014). Stimulus-response bindings in priming. Trends in Cognitive Science, 18, 376-384.

Herwig, A., \& Horstmann, G. (2011). Action-effect associations revealed by eye movements. Psychonomic Bulletin \& Review, 18, 531-537.

Herwig, A., \& Waszak, F. (2009). Intention and attention in ideomotor learning. The Quarterly Journal of Experimental Psychology, 62, 219-227.

Herwig, A., \& Waszak, F. (2012). Action-effect bindings and ideomotor learning in intention and stimulus-based actions. Frontiers in Psychology, 3, 1-18.

Hoffmann, J., Lenhard, A., Sebald, A., \& Pfister, R. (2009). Movements or targets: What makes an action in action-effect learning? The Quarterly Journal of Experimental Psychology, 62, 2433-2449.

Hoffmann, J., \& Sebald, A. (2000). Lernmechanismen zum Erwerb verhaltenssteuernden Wissens. Psychologische Rundschau, 51, 1-9.

Hommel, B. (1993). Inverting the Simon effect by intention. Psychological Research, 55, 270-279.

Hommel, B. (1998). Event files: Evidence for automatic integration of stimulus-response episodes. Visual Cognition, 5, 183-216.

Hommel, B. (2004). Event files: Feature binding in and across perception and action. Trends in Cognitive Sciences, 8, 494-500.

Hommel, B. (2009). Action control according to TEC (theory of event coding). Psychological Research, 73, 512-526.

Hommel, B., Alonso, D., \& Fuentes, L. (2003). Acquisition and generalization of action effects. Visual Cognition, 10, 965-986.

Hommel, B., \& Müsseler, J. (2006). Action-feature integration blinds to feature-overlapping perceptual events: Evidence from manual and vocal actions. The Quarterly Journal of Experimental Psychology, 59, 509-523.

Hommel, B., Müsseler, J., Aschersleben, G., \& Prinz, W. (2001). The theory of event coding (TEC): A framework for perception and action planning. Behavioral and Brain Sciences, 24, 849-878.

Hommel, B., Proctor, R. W., \& Vu, K. L. (2004). A feature-integration account of sequential effects in the Simon task. Psychological Research, 68, 1-17.

Horner, A., \& Henson, R. (2009). Bindings between stimuli and multiple response codes dominate long-lag repetition priming in speeded classification tasks. Journal of Experimental Psychology: Learning, Memory, and Cognition, 35, 757-779.

Horner, A., \& Henson, R. (2011). Stimulus-response bindings code both abstract and specific representations of stimuli: Evidence from a classification priming design that reverses multiple levels of response representation. Memory \& Cognition, 39, 1457-1471. 
Hubbard, J., Gazzaley, A., \& Morsella, E. (2011). Traditional response interference effects from anticipated action outcomes: A responseeffect compatibility paradigm. Acta Psychologica, 138, 106-110.

Humphreys, G. R., \& Buehner, M. J. (2009). Magnitude estimation reveals temporal binding at super-second intervals. Journal of Experimental Psychology: Human Perception and Performance, 35(5), 1542-1549.

Janczyk, M., Heinemann, A., \& Pfister, R. (2012). Instant attraction: Immediate action-effect bindings occur for both, stimulus-and goal-driven actions. Frontiers in Psychology, 3, 446.

Keller, P. E., \& Koch, I. (2006). Exogenous and endogenous response priming with auditory stimuli. Advances in Cognitive Psychology, 2, 269-276.

Kornblum, S., Hasbroucq, T., \& Osman, A. (1990). Dimensional overlap: Cognitive basis for stimulus response compatibility. A model and taxonomy. Psychological Review, 97, 253-270.

Kunde, W. (2001). Response-effect compatibility in manual choice reaction tasks. Journal of Experimental Psychology: Human Perception and Performance, 27, 387-394.

Kunde, W. (2003). Temporal response-effect compatibility. Psychological Research, 67, 153-159.

Logan, G. D. (1988). Toward an instance theory of automatization. Psychological Review, 95, 492-527.

Logan, G. D. (1990). Repetition priming and automaticity: Common underlying mechanisms? Cognitive Psychology, 22, 1-35.

Mayr, S., \& Buchner, A. (2006). Evidence for episodic retrieval of inadequate prime responses in auditory negative priming. Journal of Experimental Psychology: Human Perception and Performance, 32, 932-943.

Miller, J. (1987). Priming is not necessary for selective-attention failures: Semantic effects of unattended, unprimed letters. Perception \& Psychophysics, 41, 419-434.

Moeller, B., \& Frings, C. (2011). Remember the touch: Tactile distractors retrieve previous responses to targets. Experimental Brain Research, $214,121-130$.

Moeller, B., \& Frings, C. (2014a). Long term response-stimulus associations prevent distractor-response bindings. Advances in Cognitive Psychology, 10, 68-80.

Moeller, B., \& Frings, C. (2014b). Attention meets binding: Only attended distractors are used for the retrieval of event files. Attention, Perception, \& Psychophysics, 76, 959-978.

Moeller, B., Rothermund, K., \& Frings, C. (2012). Integrating the irrelevant sound - Grouping modulates the integration of irrelevant auditory stimuli into event files. Experimental Psychology, 59, 258-264.

Müsseler, J., \& Hommel, B. (1997). Blindness to response-compatible stimuli. Journal of Experimental Psychology: Human Perception and Performance, 23, 861

Müsseler, J., Wühr, P., Danielmeier, C., \& Zysset, S. (2005). Actioninduced blindness with lateralized stimuli and responses. Experimental Brain Research, 160, 214-222.

Neill, W. T. (1997). Episodic retrieval in negative priming and repetition priming. Journal of Experimental Psychology: Learning, Memory, and Cognition, 23, 1291.

Nissen, M. J., \& Bullemer, P. (1987). Attentional requirements of learning: Evidence from performance measures. Cognitive Psychology, 19, 1-32.

Pfister, R., Dignath, D., Hommel, B., \& Kunde, W. (2013). It takes two to imitate: Anticipation and imitation in social interaction. Psychological Science, 24(10), 2117-2121.

Pfister, R., Heinemann, A., Kiesel, A., Thomaschke, R., \& Janczyk, M. (2012). Do endogenous and exogenous action control compete for perception? Journal of Experimental Psychology: Human Perception and Performance, 38, 279.

Pfister, R., \& Janczyk, M. (2013). Confidence intervals for two sample means: Calculation, interpretation, and a few simple rules. Advances in Cognitive Psychology, 9, 74.

Pfister, R., Janczyk, M., Gressmann, M., Fournier, L. R., \& Kunde, W. (2014a). Good vibrations? Vibrotactile self-stimulation reveals anticipation of body-related action effects in motor control. Experimental Brain Research, 232, 847-854.

Pfister, R., Kiesel, A., \& Melcher, T. (2010). Adaptive control of ideomotor effect anticipations. Acta Psychologica, 135, 316-322.

Pfister, R., \& Kunde, W. (2013). Dissecting the response in responseeffect compatibility. Experimental Brain Research, 224, 647-655.

Pfister, R., Pfeuffer, C. U., \& Kunde, W. (2014b). Perceiving by proxy: Effect-based action control with unperceivable effects. Cognition, 132, 251-261.

Proctor, R. W., \& Reeve, T. G. (1990). Stimulus-response compatibility: An integrated perspective. North-Holland: Amsterdam.

Rescorla, R. A. (1991). Associative relations in instrumental learning: The eighteenth Bartlett memorial lecture. The Quarterly Journal of Experimental Psychology, 43B, 1-23.

Rescorla, R. A., \& Wagner, A. R. (1972). A theory of Pavlovian conditioning: Variations in the effectiveness of reinforcement and nonreinforcement. Classical Conditioning II: Current Research and Theory, 2, 64-99.

Rothermund, K., Wentura, D., \& De Houwer, J. (2005). Retrieval of incidental stimulus-response associations as a source of negative priming. Journal of Experimental Psychology: Learning, Memory, and Cognition, 31, 482-495.

Rutschmann, J., \& Link, R. (1964). Perception of temporal order of stimuli differing in sense mode and simple reaction time. Perceptual and Motor Skills, 18, 345-352.

Sato, A., \& Itakura, S. (2013). Intersubjective action-effect binding: Eye contact modulates acquisition of bidirectional association between our and others' actions. Cognition, 127, 383-390.

Schmidt, J. R., Crump, M. J. C., Cheesman, J., \& Besner, D. (2007). Contingency learning without awareness: Evidence for implicit control. Consciousness and Cognition, 16, 421-435.

Shin, Y. K., Proctor, R. W., \& Capaldi, E. J. (2010). A review of contemporary ideomotor theory. Psychological Bulletin, 136, 943-974.

Stevanovski, B., Oriet, C., \& Jolicœur, P. (2006). Symbolic-and responserelated contributions to blindness to compatible stimuli. Visual Cognition, 14, 326-350.

Stock, A., \& Hoffmann, J. (2002). Intentional fixation of behavioural learning, or how R-O learning blocks S-R learning. European Journal of Cognitive Psychology, 14, 127-153.

Thomaschke, R., Hopkins, B., \& Miall, R. C. (2012). The role of cueresponse mapping in motorvisual impairment and facilitation: Evidence for different roles of action planning and action control in motorvisual dual-task priming. Journal of Experimental Psychology: Human Perception and Performance, 38, 336.

Tipper, S. (1985). The negative priming effect: Inhibitory priming by ignored objects. The Quarterly Journal of Experimental Psychology, 37A, 571-590.

Treisman, A. M. (1960). Contextual cues in selective listening. Quarterly Journal of Experimental Psychology, 12, 242-248.

Tukey, J. (1977). Exploratory data analysis. Reading: Addison-Wesley.

Waszak, F., Hommel, B., \& Allport, A. (2004). Semantic generalization of stimulus-task bindings. Psychonomic Bulletin \& Review, 11, $1027-1033$. 